\title{
Heart failure performance measures: Eligibility and implementation in the community
}

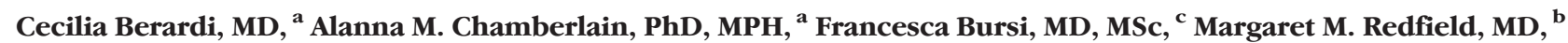
Sheila M. McNallan, MPH, ${ }^{a}$ Susan A. Weston, MS, ${ }^{a}$ Ruoxiang Jiang ${ }^{\text {a }}$ and Véronique L. Roger, MD, MPH ${ }^{\text {a,b }}$

Rochester, MN; and Modena, Italy

Background The goal of heart failure (HF) performance measures is to improve quality of care by assessing the implementation of guidelines in eligible patients. Little is known about the proportion of eligible patients and how performance measures are implemented in the community.

Methods We determined the eligibility for and adherence to performance measures and $\beta$-blocker therapy in a community-based cohort of hospitalized HF patients from January 2005 to June 2011.

Results All of the $465 \mathrm{HF}$ inpatients (median age 76 years, $48 \%$ men) included in the study received an ejection fraction assessment. Only 164 had an ejection fraction $<40 \%$ thus were candidates for $\beta$-blocker and angiotensin converting enzyme (ACE) inhibitors or angiotensin receptor blocker (ARB) therapy. Considering absolute contraindications, 99 patients were eligible to receive ACE inhibitors/ARB, and 162 to receive $\beta$-blockers. Among these, $85 \%$ received ACE inhibitors/ARBs and $91 \%$ received $\beta$-blockers. Among the 261 individuals with atrial fibrillation, 89 were eligible for warfarin and $54 \%$ received it. Of 52 current smokers, $69 \%$ received cessation counseling during hospitalization.

Conclusion In the community, among eligible hospitalized HF patients, the implementation of performance measures can be improved. However, as most patients are not candidates for current performance measures, other approaches are needed to improve care and outcomes. (Am Heart J 2013;166:76-82.)

Heart failure (HF) affects nearly 6 million people in the United States. ${ }^{1}$ Due to its high hospitalization rate and associated morbidity, HF is one of the most costly health related conditions in the United States. ${ }^{1}$ Therefore, it represents a major target to optimize the quality of care and improve outcomes.

In 2005, the American College of Cardiology Foundation/ American Heart Association evaluated existing and emerging quality measures for HF according to validity, reliability, and feasibility criteria. As a result, a set of inpatient performance measures assessing relevant dimensions of care in hospitalized patients with HF was created. ${ }^{2}$

From the a Department of Health Sciences Research, Mayo Clinic, Rochester, MN, ${ }^{b}$ Division of Cardiovascular Diseases, Mayo Clinic, Rochester, MN, and 'Division of Cardiology, University Hospital of Modena, Modena, Italy.

This work was supported by grants from the National Heart, Lung, and Blood Institute (RO1 HL72435) and the National Institute on Aging (RO1 AG034676) of the National Institutes of Health. Dr. Roger is an Established Investigator of the American Heart Association. The funding sources played no role in the design, conduct, or reporting of this study and the content does not necessarily represent the official views of the National Institutes of Health Submitted November 1, 2012; accepted March 14, 2013.

Reprint requests: Véronique L. Roger, MD, MPH, Department of Health Sciences Research Mayo Clinic, 200 First St. SW, Rochester, MN 55095.

E-mail: roger.veronique@mayo.edu

0002-8703/\$ - see front matter

(C) 2013, Mosby, Inc. All rights reserved.

http://dx.doi.org/10.1016/j.ahj.2013.03.008
Subsequently, performance data have been publicly reported and pay-for-performance programs have been created to foster their implementation in clinical practice. Despite the increasing adherence to in-hospital care performance measures, a consistent improvement in outcome has not been noted, ${ }^{3-5}$ exposing a potential gap between quality measures and clinical practice.

The guidelines report inclusion and exclusion criteria for each measure, identifying a subset of eligible patients within the general HF population. Previous studies on myocardial infarction performance measures demonstrated that a considerable number of patients with myocardial infarction are not eligible for such measures due to contraindications. ${ }^{6}$ To the best of our knowledge, this topic has not been specifically addressed in HF patients. Our goal was to evaluate eligibility for and adherence to performance measures among a cohort of patients hospitalized with $\mathrm{HF}$ between 2005 and 2011. We tested the hypothesis that, in the community, the eligibility for performance measures is limited to a small number of individuals.

\section{Methods}

\section{Study setting}

This cohort study was conducted in southeastern Minnesota among a community population of hospitalized individuals with incident and prevalent HF. 
for patients with LVSD, without contraindications for both ACE inhibitors and ARBs.

Trovided County, the relatively small number of health care providers (mainly Mayo Clinic and Olmsted Medical Center) and the use of a comprehensive medical records system ${ }^{7}$ enabled us to extensively capture patients' characteristics, past medical history, and relevant aspects of HF management during hospitalization. Through the record linkage system of the Rochester Epidemiology Project, ${ }^{8}$ data were also captured for Dodge and Fillmore County residents who were hospitalized at an Olmsted County hospital.

This study was approved by the Mayo Clinic and Olmsted Medical Center Institutional Review Boards.

\section{Identification of the study cohort}

Potential HF patients were identified using natural language processing of the electronic medical record as previously described. ${ }^{9}$ HF diagnoses were validated by nurse abstractors using the Framingham criteria. ${ }^{10}$ We included all individuals aged 18 years or older who were residents of Olmsted, Fillmore, or Dodge County, MN, and admitted to an Olmsted County hospital between January 2005 and June 2011 with first-ever (incident) or primary admission diagnosis (prevalent) of HF. Patients who died during hospitalization were excluded. Written informed consent was obtained from all participants prior to enrollment.

\section{Clinical data collection}

The following patient characteristics during the hospitalization were obtained from the medical records. Body mass index was calculated as weight $(\mathrm{kg})$ divided by height $(\mathrm{m})$ squared. Serum creatinine was used to estimate the glomerular filtration rate using the MDRD study equation. ${ }^{11}$ Left ventricular ejection fraction (\%) was obtained from the echocardiogram performed during the hospitalization that was closest to HF diagnosis.

Nurse abstractors obtained past medical history on comorbidities and cardiovascular risk factors from the medical records. Individuals with a clinical diagnosis, systolic blood pressure $\geq 140 \mathrm{~mm} \mathrm{Hg}$, diastolic blood pressure $\geq 90 \mathrm{~mm} \mathrm{Hg}$, or use of an antihypertensive medication ${ }^{12}$ were defined as having hypertension. Hyperlipidemia was defined using the National Cholesterol Education Program guidelines ${ }^{13}$ or use of medications for hyperlipidemia. The World Health Organization criteria

${ }^{14}$ were used to define anemia (hemoglobin $<13 \mathrm{mg} / \mathrm{dL}$ in men and $<12 \mathrm{mg} / \mathrm{dL}$ in women) using the hemoglobin level closest to HF diagnosis date. The American Diabetes Association criteria ${ }^{15}$ were used to identify prevalent diabetes. Comorbidity was scored using the Charlson comorbidity index. ${ }^{16}$

\section{Performance measures}

To assess quality of care among this population of hospitalized HF patients, we analyzed 5 quality performance measures. Four of them were recommended by the American College of Cardiology Foundation/American Heart Association for HF inpatients ${ }^{2}$ and were defined as follows:

1) Evaluation of left ventricular systolic function through documented assessment of ejection fraction (EF) before or during hospitalization, or planned after discharge. Left ventricular systolic dysfunction (LVSD) was defined as $\mathrm{EF}<40 \%$.

2) Angiotensin converting enzyme (ACE) inhibitor or angiotensin receptor blocker (ARB) therapy at discharge
3) Anticoagulant therapy at discharge for HF patients with atrial fibrillation (AF) without warfarin contraindications.

4) Adult smoking cessation advice/counseling for current smokers.

In addition, since $\beta$-blocker therapy is mentioned in the guidelines, ${ }^{17}$ is frequently used in clinical practice, and has been associated with better outcomes in hospitalized HF patients, ${ }^{3}$ we also assessed prescription of $\beta$-blockers in HF patients with LVSD.

\section{Eligibility for and adherence to performance measures}

We defined "candidates" for performance measures as patients presenting the essential characteristics that, according to guidelines, ${ }^{2,17}$ justify the application of the measure considered. Whereas each patient with a HF diagnosis qualified for an evaluation of left ventricular function, only individuals with $\mathrm{EF}<40 \%$ were candidates for ACE inhibitors, ARBs, or $\beta$ blocker therapy. A previous diagnosis of AF in the medical record identified candidates for anticoagulation therapy. Current smoking (upon admission or anytime during the previous six months, according to the definition in our practice) identified candidates for cessation counseling.

To assess "eligible" patients, we excluded individuals with contraindications for a given performance measure. There is no contraindication to EF measurement or smoking cessation counseling. For the remaining quality measures, we used the International Classification of Diseases, Ninth revision (ICD9) codes to define "eligible" patients (Table I), using the ICD-9 codes suggested by Bridges to Excellence Congestive Heart Failure Care Link $^{18}$ to identify contraindications for ACE inhibitors, ARBs, and $\beta$-blockers, along with a list of ICD-9 codes as previously published by Glazer et $\mathrm{al}^{19}$ to identify contraindications to warfarin. In addition, we distinguished between "absolute" and "relative" contraindications to determine "eligible" patients for $\beta$-blockers. In particular, we considered as relative contraindications a history of chronic obstructive pulmonary disease (ICD-9 codes 491.20, 491.21, $491.22,493.20,493.21,493.22,496)$ or asthma (ICD-9 codes 493.00, 493.01, 493.02, 493.10, 493.11, 493.12, 493.80, 493.81, 493.82, 493.90, 493.91, 493.92) and a history of intraventricular pacemaker (426.13, 426.2, 426.4, 426.50, 426.51, 426.52, 426.53, 426.54, 426.6, 426.7)

Finally, we assessed the proportion of individuals who received the intervention prescribed by each performance measure among eligible patients. Discharge medications and smoking cessation advice or counseling during hospitalization were abstracted from medical records. Patients who were offered but refused the smoking cessation counseling were considered adherent to the performance measure.

\section{Statistical analysis}

All analyses were conducted using SAS statistical software, version 9.2 (SAS Institute Inc, Cary, NC). Characteristics of the HF patients were described using median (quartile 1 to quartile 3 ) of the distribution for continuous variables and number (percentage) for categorical variables. For each performance measure, we first identified the candidates (eg, only those with conduction defects other than atrioventricular block, with no 
Table I. Contraindications for angiotensin converting enzyme inhibitor or angiotensin receptor blocker, $\beta$-blockers and anticoagulant therapy

Performance measure

ACE inhibitor or $A R B$

Allergy or intolerance (995.0 and E942.9, 995.1 and E942.9, 995.27 and E942.9, 995.29 and E942.9)

$\mathrm{n}=0$

Moderate/severe aortic stenosis (440.1, 395.0, 396.0, 396.2, 396.8, 425.1, 747.22)

$\mathrm{n}=28$

Previous severe adverse reactions (Angioedema: 995.1 or Anuric renal failure: V56.0, V56.8, 39.95, 54.98, $\quad \mathrm{n}=50$

788.5, 586, 403.01, 403.11, 403.91, 404.02, 404.03, 404.12, 404.13, 404.92, 404.93, 584.5-584.9,

$585.5,585.6)$

Pregnancy (V22.0-V23.9)

Anticoagulant therapy Allergy or intolerance (995.0 and E934.2, 995.1 and E934.2, 995.27 and E934.2, 995.29 and E934.2) Pregnancy (V22.0-V23.9)

Previous gastrointestinal/genitourinary hemorrhage $(578 . x, 531.0,531.2,531.4,531.6,532.0,532.2$,

$\mathrm{n}=0$

$\mathrm{n}=0$

$\mathrm{n}=67$ $532.4,532.6,533.0,533.2,533.4,533.6,534.0,534.2,534.4,534.6,535 . x 1,530.82,456.0,456.2$, $569.3,596.7)$

Prior intracranial hemorrhage: (430.x, 431.x, 432.0, 432.1, 432.9, 851, 852.xx, 853.xx,854.xx)

Prior other hemorrhage $(362.81,459.0,786.3)$

Prior cirrhosis/ hepatitis (070.2-070.9, 570, 571.x, 572.2, 572.3, 572.4, 572.8)

Hemorrhagic tendencies or blood dyscrasias $(286.0,286.5,286.6,286.7,286.9,287.3,287.4,287.5$,

287.8, 287.9, 289.9)

Alcoholism or drug abuse (303.x, 304.x,305.x [excluding 305.1])

Perceived barriers to compliance (295.x-298.x, V60.0-V60.4, V15.81)

Predisposition to falls (290.x-294.x. 331.0, 331.1, 333.4, 345.x, 347, 458.0, 780.2, 780.3,

E884.2-E884.6, 733.1)

Prior cerebral aneurism (407.3)

Beta-blockers

Allergy/intolerance (995.0 and E942.9, 995.1 and E942.9, 995.27 and E942.9, 995.29 and E942.9)

Marked bradycardia abstracted from electrocardiogram (HR $<50 \mathrm{bpm})$

AV block 2nd type 2/3rd degree and no pace maker $(426.0,426.12)$

$\mathrm{n}=8$

$\mathrm{n}=28$

$\mathrm{n}=21$

$\mathrm{n}=55$

$\mathrm{n}=8$

$\mathrm{n}=28$

$\mathrm{n}=67$

$\mathrm{n}=0$

$\mathrm{n}=0$

$\mathrm{n}=0$

$\mathrm{n}=2$

*Contraindications groups were not mutually exclusive, multiple contraindications might be present in the same patient.

Table II. Characteristics of hospitalized HF patients ( $N=465)$

Socio-demographic variables

Age (years)

Male

Cardiovascular risk factors

Hypertension

Hyperlipidemia

Current smoking

Diabetes mellitus

Body mass index $\left(\mathrm{kg} / \mathrm{m}^{2}\right)$

Comorbidities

Atrial fibrillation/flutter

Myocardial infarction

Charlson comorbidity index

0

1-2

$\geq 3$

Estimated glomerular filtration rate

$\left(\mathrm{mL} / \mathrm{min}\right.$ per $\left.1.73 \mathrm{~m}^{2}\right)$

Anemia

Heart failure characteristics

Ejection fraction (\%)

$76.0(65.0-84.0)$ $225(48.4)$

$409(88.0)$

$354(76.1)$

$52(11.2)$

$161(34.6)$

$29.6(25.0-34.8)$

$261(56.1)$

$119(25.7)$

$51(11.0)$

$122(26.4)$

$290(62.6)$

$56.5(42.8-72.1)$

$265(57.1)$

$51.0(33.8-62.0)$

Values are presented as $n(\%)$ for categorical variables and median (Q1-Q3) for continuous variables.

LVEF were candidates for ACE inhibitors/ARB and only patients with AF were candidates for warfarin). Then, we reported the number of patients who were eligible for the performance measure after deleting those with contraindications to the performance measure. Finally, we reported the adherence to each performance measure by describing the number (percent-

\section{Figure 1}

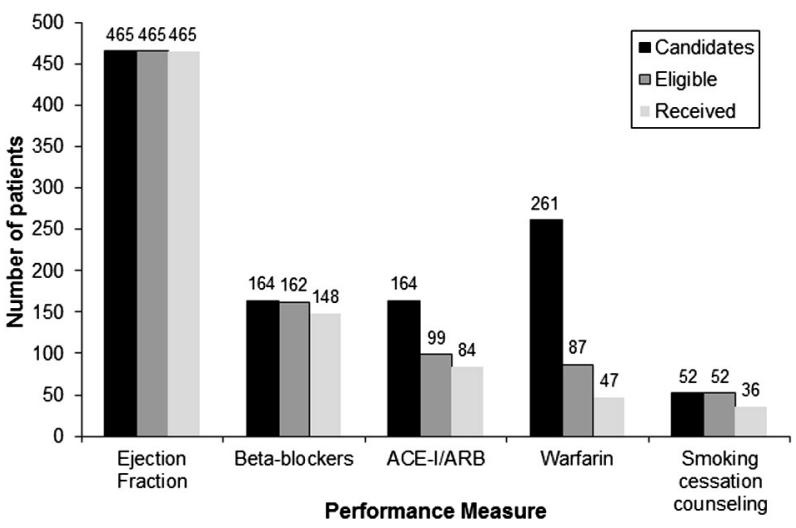

Number of heart failure patients who were candidates for and eligible to receive performance measures.

age) who received the performance measure out of the total eligible for each measure. In addition, we provided the number of patients eligible for 1, 2, 3, 4, or all 5 performance measures.

This work was supported by grants from the National Heart, Lung, and Blood Institute (R01 HL72435) and the National Institute on Aging (R01 AG034676) of the National Institutes of Health. Dr. Roger is an Established Investigator of the American Heart Association. The funding sources played no role in the 
Figure 2

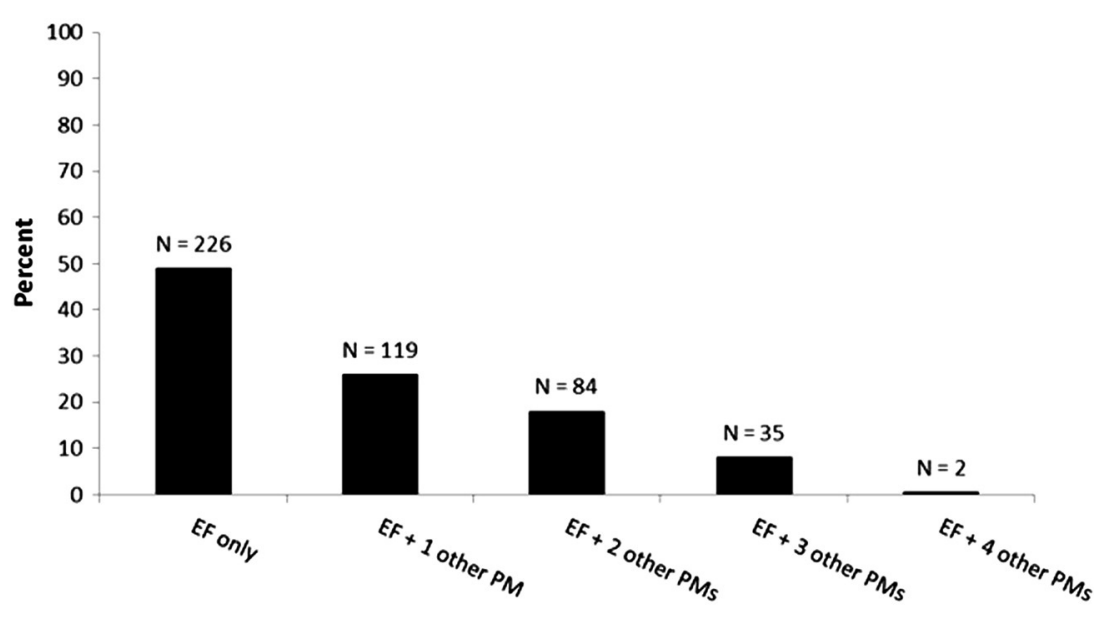

Percent of Patients Eligible for Performance Measures

Number and percentage of patients eligible for one or more performance measures.

design, conduct, or reporting of this study and the content does not necessarily represent the official views of the National Institutes of Health. The authors are solely responsible for the design and conduct of this study, all study analyses, the drafting and editing of the paper and its final contents.

\section{Results}

Between January 2005 and June 2011, we enrolled 647 hospitalized patients with HF. Twenty of them died during hospitalization and were therefore excluded from the analysis as no discharge information was available in order to assess performance measures; of the remaining patients, we excluded 160 individuals who were admitted to the hospital with a primary diagnoses other than $\mathrm{HF}$ and 2 individuals whose electrocardiogram was not available in the medical records. Overall, we assessed performance measures in 465 inpatients (median age [1st quartile, 3rd quartile]: $76[65,84]$ years, $48 \%$ men). The patient characteristics are summarized in Table II. There was a high prevalence of comorbidities; $63 \%$ of the cohort had a score of 3 or more according to Charlson index. Median ejection fraction (1st quartile, 3 rd quartile) was $51 \%(33.8,62.0)$.

Applicability of performance measures in the community According to guidelines, all incident and prevalent $\mathrm{HF}$ cases were eligible for left ventricular systolic function evaluation during hospitalization (Figure 1). All 465 patients fulfilled this first performance measure, but only 164 had documented systolic dysfunction, thereby meeting criteria for prescription of ACE inhibitor or ARB therapy, as well as $\beta$-blocker therapy. Sixty-five of the 164 candidates with documented left ventricular systolic dysfunction had absolute contraindications for both ACE inhibitors and ARBs leaving only 99 eligible patients. For $\beta$ blocker therapy, 2 patients were excluded due to absolute contraindications, leaving 162 eligible patients. If we considered relative contraindications for $\beta$-blockers, the number of eligible patients decreased to 105 . There were $261 \mathrm{HF}$ patients with a history of AF who were candidates to receive anticoagulation therapy. After excluding those with contraindications to anticoagulation therapy, only 87 patients were eligible to receive warfarin. Finally, 52 patients were current smokers, and thus were candidates for smoking cessation counseling.

As shown in Figure 2, about half of the study population was eligible only for left ventricular systolic function evaluation and was not eligible for any other performance measure. The number of eligible patients progressively decreased when considering more than one performance measure.

Adherence to performance measures in the community Adherence to performance measures is shown in Figure 1. EF was measured in all patients during hospitalization, thus $100 \%$ of the study population received left ventricular systolic function evaluation. ACE inhibitors or ARBs were prescribed to $85 \%$ of eligible patients at hospital discharge. In addition, 91\% of eligible patients received $\beta$-blocker therapy; after excluding relative contraindications for $\beta$-blockers from the denominator, 93\% of eligible patients were prescribed the medication. Anticoagulation with warfarin was prescribed to $54 \%$ of patients with concomitant $\mathrm{AF}$ and no contraindications. Finally, among current smokers, 69\% received cessation counseling during hospitalization. Of these, 8 patients were offered but refused the counseling. 


\section{Discussion}

Herein, we report that, in the community, only a small proportion of patients hospitalized with HF are candidates for performance measures and $\beta$-blocker therapy. Furthermore, a lower proportion of patients are eligible for performance measures due to contraindications. Adherence to the guidelines varied by measure. The assessment of EF was universal, and the implementation of $\beta$-blocker therapy was more than adequate among patients who could receive it. Conversely, ACE inhibitors or ARB therapy, and especially smoking cessation counseling and anticoagulant therapy in eligible patients can be improved.

\section{Applicability of performance measures in the community}

Most studies on performance measures focused on the effect of their implementation on outcome. . $^{4,20-23}$ However, data on eligibility for each performance measure are sparse. In our cohort, only 35\% of individuals had an $\mathrm{EF}<40 \%$ and, therefore, were candidates to receive ACE inhibitors or ARB therapy and $\beta$-blockers according to guidelines. After excluding patients with contraindications, only $21 \%$ of the total population was eligible for ACE inhibitors or ARBs. These results expose a considerable gap between the quality measures and the real case mix of patients with HF. Similarly, anticoagulation is indicated when concomitant AF is present. Moreover, warfarin has multiple contraindications that substantially limit eligibility, especially considering the increasing age and prevalence of comorbidities in HF. Taken together, these observations account for the exclusion of over $80 \%$ of our patients from the eligible group for anticoagulation.

Our results for ACE inhibitors/ARBs and warfarin differ substantially from previous studies. For example, Intermountain Healthcare, where $88 \%$ of patients were eligible for ACE inhibitors or ARB; ${ }^{21}$ importantly, more than 20\% of patients did not undergo systolic function assessment which may partially explain the higher percentage of eligible individuals for ACE inhibitors or ARB therapy in this report. In addition, a previous study conducted on a cohort of individuals with impaired EF reported that only $13 \%$ of the patients had a documented contraindication for ACE inhibitors and ARBs. ${ }^{24}$ Finally, in the OPTIMIZE-HF registry, the number of patients suitable to be prescribed warfarin was considerably higher compared to what was found in our population. ${ }^{20}$ Differences observed between the aforementioned studies and our study may partly be due to the inclusion of a more broad spectrum of contraindications in our analysis or to the limited capability of ICD-9 codes to document the decision making process of the caring physician. We utilized a set of codes previously published to document contraindications in a "rewarding quality" program, but we acknowledge that codes do not account for the physician's risk benefit evaluation, and thus therapeutic decision, for the specific case. Nevertheless, previous literature seems to consistently show that a relevant proportion of HF patients presents with contraindications for medications included in current performance measures.

Finally, there were few active smokers in our study. Thus, the applicability of the measure focused on smoking cessation counseling was quite limited in our population. These findings resonate with results from previous studies. ${ }^{20,21}$

Taken together, these data show that all 5 of the performance measures apply to a minority of patients with HF; in fact, only 2 of 465 patients in our communitybased study were eligible for all 5 performance measures. While we acknowledge that two of the five considered performance measures are only applicable to patients with LVSD, our goal is to provide insight on the "realworld" HF population, exposing a possible gap between the latter and the patients for whom the quality measurements are relevant.

As underscored by our results, the lack of specific treatment and hence guidelines for $\mathrm{HF}$ with preserved $\mathrm{EF}$ leads to the exclusion of more than half of the HF population from performance measures. This creates a challenge for quality improvement strategies such as public reports or pay-for-performance programs. To provide the public with real evidence regarding inpatient quality of care, the proportion of non-eligible individuals and their characteristics should be reported. This would also guide future research in the development and implementation of performance measures.

Adherence to performance measures in the community

In our cohort, LV systolic function assessment was universal, higher than the previously reported range from $79 \%$ to $87 \% .^{20,21}$ This may reflect the practice of a large teaching hospital as Heidenreich et al have previously reported. $^{25}$

We found $85 \%$ adherence to ACE inhibitors or ARBs in our study, in line with reports from prospective registries. ${ }^{20,21,25}$ However, $\beta$-blockers were prescribed to a higher number of patients in our population than in OPTIMIZE-HF registry, reflecting the different time period and the widespread clinical adoption of $\beta$ blockers in HF. ${ }^{20}$

Conversely, smoking cessation counseling was prescribed only to $69 \%$ of our population and only half of the eligible population received anticoagulation therapy. Smoking cessation counseling can be incompletely ascertained, as some patients may have received smoking cessation advice without formal documentation in the medical record and this could have led to an underestimation of adherence. Similarly, the decision to prescribe warfarin, due to the medication side effects and interactions, is based on a comprehensive evaluation of the 
patient where the physician's judgment has a central role, and this process is difficult to capture by automated queries. $^{26}$ On the other hand, if we consider anticoagulation therapy, our data resonate with previous studies on patients with HF and AF showing a lower than expected use of warfarin in eligible patients. ${ }^{20,27}$

\section{Updated set of inpatient performance measures}

Recently, the American College of Cardiology Foundation, the American Heart Association, and the American Medical Association-Physician Consortium for Performance Improvement have modified and partially integrated inpatient performance measures. ${ }^{28}$

The new set includes a total of four performance measures: two are applicable to the entire HF population (left ventricular systolic function and post-discharge follow-up appointment) and two are applicable to patients with LV systolic dysfunction only ( $\beta$-blocker therapy and ACE-inhibitor or ARB therapy). These changes will simplify the assessment and documentation of quality of care in HF patients, while reflecting the most recent knowledge on the management of HF. However, whether the number of patients identified as "candidates" will change with the implementation of these new measures is uncertain given the presentation of $\mathrm{HF}$ in the community as documented herein.

\section{Limitations and strengths}

Some limitations must be acknowledged while interpreting our results. First, we relied on ICD-9 codes to identify contraindications for some of the performance measures and, thus, we cannot exclude misclassification of eligibility for ACE inhibitors/ARBs, $\beta$-blockers, or anticoagulation therapy. Second, delivery of discharge instructions could not be assessed from the medical record.

Third, the number of eligible individuals for some of the performance measures precluded assessing clinical outcomes according to eligibility. Finally, the southeastern Minnesota population consists mainly of whites, which may affect the generalizability of our results; however, our population is representative of Minnesota and the Upper Midwest region of the US. ${ }^{29}$

Our study also has distinct strengths. We are not aware of another study addressing eligibility among a community cohort of patients hospitalized with HF. Doing so in the community is essential to offer optimal clinical relevance and reflect the real case mix and management of HF patients.

\section{Conclusions}

In the community, inpatient performance measures are only applicable to a small number of individuals, thus limiting the assessment of quality of care in the HF population.
Among eligible patients, the implementation of performance measures varies by measure. While the percentage of patients meeting the measure is high for LV systolic function assessment, and $\beta$-blockers and ACE inhibitor/ARB prescription at dismissal, smoking cessation counseling and anticoagulation are underutilized in eligible patients.

\section{Acknowledgements}

We thank Annette L. McNallan, RN, Kay A. Traverse, $\mathrm{RN}$, and Deborah S. Russell for their study support.

\section{Disclosures}

None.

\section{References}

1. Roger VL, Go AS, Lloyd-Jones DM, et al. Heart disease and stroke statistics-2012 update: a report from the American Heart Association. Circulation 2012;125(1):e2-e220.

2. Bonow RO, Bennett $S$, Casey Jr DE, et al. ACC/AHA clinical performance measures for adults with chronic heart failure: a report of the American College of Cardiology/American Heart Association Task Force on Performance Measures (Writing Committee to Develop Heart Failure Clinical Performance Measures) endorsed by the Heart Failure Society of America. J Am Coll Cardiol 2005;46(6):1 144-78.

3. Fonarow GC. Performance measures for patients hospitalized with heart failure: are they predictive of clinical outcomes? Congest Heart Fail 2007;13(6):342-6.

4. Patterson ME, Hernandez AF, Hammill BG, et al. Process of care performance measures and long-term outcomes in patients hospitalized with heart failure. Med Care 2010;48(3):210-6.

5. Hernandez AF, Fonarow GC, Liang $L$, et al. The need for multiple measures of hospital quality: results from the Get with the GuidelinesHeart Failure Registry of the American Heart Association. Circulation 2011;124(6):712-9.

6. Bernheim SM, Wang Y, Bradley EH, et al. Who is missing from the measures? Trends in the proportion and treatment of patients potentially excluded from publicly reported quality measures. Am Heart J 2010;160(5):943-50 e 1-5.

7. St Sauver JL, Grossardt BR, Yawn BP, et al. Use of a medical records linkage system to enumerate a dynamic population over time: the Rochester epidemiology project. Am J Epidemiol 201 1;173(9):1059-68.

8. Melton III $\mathrm{L}$. History of the Rochester Epidemiology Project. Mayo Clin Proc 1996;71(3):266-74

9. Bursi F, Weston SA, Redfield MM, et al. Systolic and diastolic heart failure in the community. JAMA 2006;296(18):2209-16.

10. Ho KK, Anderson KM, Kannel WB, et al. Survival after the onset of congestive heart failure in Framingham Heart Study subjects. Circulation 1993;88(1):107-15.

11. Levey AS, Coresh J, Greene T, et al. Using standardized serum creatinine values in the modification of diet in renal disease study equation for estimating glomerular filtration rate. Ann Intern Med 2006; 145(4):247-54.

12. Chobanian AV, Bakris GL, Black HR, et al. The Seventh Report of the Joint National Committee on Prevention, Detection, Evaluation, and Treatment of High Blood Pressure: the JNC 7 report. JAMA 2003; 289(19):2560-72. 
13. Third Report of the National Cholesterol Education Program (NCEP) Expert Panel on Detection, Evaluation, and Treatment of High Blood Cholesterol in Adults (Adult Treatment Panel III) final report. Circulation 2002;106(25):3143-421.

14. Izaks GJ, Westendorp RG, Knook DL. The definition of anemia in older persons. JAMA 1999;281(18):1714-7.

15. Standards of medical care in diabetes-2006. Diabetes Care 2006; 29(Suppl 1):S4-S42.

16. Charlson ME, Pompei $P$, Ales KL, et al. A new method of classifying prognostic comorbidity in longitudinal studies: development and validation. J Chronic Dis 1987;40(5):373-83.

17. Jessup M, Abraham WT, Casey DE, et al. 2009 focused update: ACCF/AHA Guidelines for the Diagnosis and Management of Heart Failure in Adults: a report of the American College of Cardiology Foundation/American Heart Association Task Force on Practice Guidelines: developed in collaboration with the International Society for Heart and Lung Transplantation. Circulation 2009;1 19(14):1977-2016.

18. Excellence Bt. Congestive heart failure care link physician assessment.

19. Glazer NL, Dublin S, Smith NL, et al. Newly detected atrial fibrillation and compliance with antithrombotic guidelines. Arch Intern Med 2007;167(3):246-52.

20. Fonarow GC, Abraham WT, Albert NM, et al. Association between performance measures and clinical outcomes for patients hospitalized with heart failure. JAMA 2007;297(1):61-70.

21. Kfoury AG, French TK, Horne BD, et al. Incremental survival benefit with adherence to standardized heart failure core measures: a performance evaluation study of 2958 patients. J Card Fail 2008; 14(2):95-102.

22. VanSuch $M$, Naessens JM, Stroebel RJ, et al. Effect of discharge instructions on readmission of hospitalised patients with heart failure: do all of the Joint Commission on Accreditation of Healthcare Organizations heart failure core measures reflect better care? Qual Saf Health Care 2006;15(6):414-7.

23. Jha AK, Orav EJ, Li Z, et al. The inverse relationship between mortality rates and performance in the Hospital Quality Alliance measures. Health Aff (Millwood) 2007;26(4): 1 104-10.

24. Peterson PN, Rumsfeld JS, Liang L, et al. Treatment and risk in heart failure: gaps in evidence or quality? Circ Cardiovasc Qual Outcomes 2010;3(3):309-15.

25. Heidenreich PA, Zhao X, Hernandez AF, et al. Patient and hospital characteristics associated with traditional measures of inpatient quality of care for patients with heart failure. Am Heart J 2012; 163(2):239-45 e3.

26. Rosenman MB, Simon TA, Teal E, et al. Perceived or actual barriers to warfarin use in atrial fibrillation based on electronic medical records. Am J Ther 2012;19(5):330-7.

27. Piccini JP, Hernandez AF, Zhao X, et al. Quality of care for atrial fibrillation among patients hospitalized for heart failure. J Am Coll Cardiol 2009;54(14):1280-9.

28. Bonow RO, Ganiats TG, Beam CT, et al. ACCF/AHA/AMA-PCPI 2011 Performance Measures for Adults With Heart Failure: A Report of the American College of Cardiology Foundation/American Heart Association Task Force on Performance Measures and the American Medical Association-Physician Consortium for Performance Improvement. J Am Coll Cardiol 2012;59(20): 1812-32.

29. St Sauver JL, Grossardt BR, Leibson CL, et al. Generalizability of epidemiological findings and public health decisions: an illustration from the Rochester Epidemiology Project. Mayo Clin Proc 2012; 87(2):151-60. 